\title{
Molecular Mapping of Neutral Lipids using Silicon Nanopost Arrays and TIMS Imaging Mass
}

\section{Spectrometry}

Jarod A. Fincher ${ }^{1,2}$, Katerina V. Djambazova ${ }^{1,3}$, Dustin R. Klein ${ }^{1,2}$, Martin Dufresne ${ }^{1,2}$, Lukasz G. Migas ${ }^{4}$, Raf Van de Plas ${ }^{1,2,4}$, Richard M. Caprioli ${ }^{1,2,3,5,6}$, and Jeffrey M. Spraggins ${ }^{1,2,3^{*}}$

${ }^{1}$ Mass Spectrometry Research Center, Vanderbilt University, $46521^{\text {st }}$ Ave S \#9160, Nashville, TN 37235

${ }^{2}$ Department of Biochemistry, Vanderbilt University, 607 Light Hall, Nashville, TN 37205, USA

${ }^{3}$ Department of Chemistry, Vanderbilt University, 7330 Stevenson Center, Station B 351822, Nashville, TN 37235, USA

${ }^{4}$ Delft Center for Systems and Control, Delft University of Technology, 2628 CD Delft, The Netherlands ${ }^{5}$ Department of Pharmacology, Vanderbilt University, 442 Robinson Research Building, 2220 Pierce Avenue, Nashville, TN 37232, USA

${ }^{6}$ Department of Medicine, Vanderbilt University, $46521^{\text {st }}$ Ave S \#9160, Nashville, TN 37235, USA 


\begin{abstract}
We demonstrate the utility of combining silicon nanopost arrays (NAPA) and trapped ion mobility imaging mass spectrometry (TIMS IMS) for high spatial resolution and specificity mapping of neutral lipid classes in tissue. Ionization of neutral lipid species such as triglycerides (TGs), cholestryl esters (CEs), and hexosylceramides (HexCers) from biological tissues has remained a challenge for imaging applications. NAPA, a matrix-free laser desorption ionization (LDI) substrate, provides enhanced ionization efficiency for the abovementioned neutral lipid species, providing complementary lipid coverage to matrix-assisted laser desorption ionization (MALDI). The combination of NAPA and TIMS IMS enables imaging of neutral lipid species at $20 \mu \mathrm{m}$ spatial resolution while also increasing molecular coverage greater than 2 -fold using gas-phase ion mobility separations. This is a significant improvement with respect to sensitivity, specificity, and spatial resolution compared to previously reported imaging studies using NAPA alone. Improved specificity for neutral lipid analysis using TIMS IMS was shown using rat kidney tissue to separate TGs, CEs, HexCers, and phospholipids (PLs) into distinct ion mobility trendlines. Further, this technology allowed for the separation of isomeric species, including mobility resolved isomers of $\operatorname{Cer}(\mathrm{d} 42: 2)(\mathrm{m} / \mathrm{z}$ 686.585 ) with distinct spatial localizations measured in rat kidney tissue section.
\end{abstract}

\title{
Introduction
}

Imaging mass spectrometry (IMS) enables the spatial mapping of biomolecules such as proteins ${ }^{1,2}$, peptides $^{3,4}$, lipids $^{5,6}$ and metabolites ${ }^{7,8}$ in biological tissues with high chemical specificity and without the need for target specific reagents. IMS has been widely adopted in fields like molecular histology for differentiation of cancerous and noncancerous tissues ${ }^{9-11}$, as well as the pharmaceutical industry for analyzing the distribution of drugs and their metabolites in tissues. ${ }^{12,} 13$ Matrix-assisted laser desorption ionization (MALDI), which requires deposition of a UV-absorbing matrix onto the tissue before analysis, is currently the method-of-choice for most IMS applications due to its unmatched molecular coverage and sensitivity. In the MALDI IMS experiment, samples are thinly sectioned and mounted onto a conductive substrate prior to coating with a matrix that assists in desorption and ionization of endogenous molecules. lons are generated by laser irradiation and detected by mass spectrometry whereby a mass spectrum is produced at each ablated position (pixel). The spatial resolution of the image is defined by the size of the laser spot at the tissue surface, the spacing between pixels in the sampled array (i.e. pitch), and factors arising from sample preparation (e.g. matrix crystal size for MALDI). Imaging experiments are routinely performed at $20 \mu \mathrm{m}$ or greater with commercial platforms ${ }^{14}$ and can achieve resolutions of $\leq 5 \mu \mathrm{m}$ with modifications to the laser optics ${ }^{15,16}$, implementation of transmission geometry. ${ }^{17}$ In addition, recently 
developed methods for improving sensitivity have been implemented such as laser-induced postionization (MALDI-2). ${ }^{18,19}$

While MALDI has been shown to be effective in ionizing a broad range of molecular classes, it typically does not adequately ionize neutral lipids such as triglycerides (TGs), cholesteryl esters (CEs), and hexosylceramides (HexCers) from complex tissue samples due to ion suppression from phospholipids. ${ }^{20-22}$ To address this issue, alternative methods have been developed including silver or gold nanoparticle coatings ${ }^{20,21}$, salt doping of the MALDI matrix ${ }^{22}$, and MALDI- $2^{23}$ to enhance ionization efficiency for neutral lipids. Another approach is the use of silicon nanopost arrays (NAPA), a matrix-free LDI imaging substrate, that has been shown to selectively ionize neutral lipids with enhanced ionization efficiency. ${ }^{24,}{ }^{25}$ For example, bacterially infected and control human skin tissue samples were able to be differentiated by NAPA-LDI based on differences in neutral lipid profiles including TGs and HexCers. ${ }^{26}$ As a matrix-free approach, NAPA simplifies sample preparation steps and helps expand the diversity of lipid species that can be investigated for imaging applications.

In light of the inherent complexity and vast diversity of lipid species present within biological systems, the development of analytical platforms capable of resolving isobaric and isomeric lipid species while maintaining their respective spatial integrities is critical for fully characterizing the molecular drivers of biology in healthy and diseased tissues. ${ }^{27-29}$ Mass analyzers such as the Orbitrap and Fourier transform ion cyclotron resonance (FT-ICR) with their unparalleled high mass accuracy and mass resolving power capabilities have been instrumental in resolving isobaric lipid species for both routine lipidomic studies and imaging applications. However, these mass analyzers cannot differentiate isomeric lipid species of exact mass. For example, lipid species that have the same exact mass can differ in their fatty acid chain configuration or fatty acid chain location (sn positional isomers). While isomeric lipid species have often been resolved by implementing liquid chromatography (LC) separations prior to mass spectrometry analysis, this requires homogenization of the sample and the timescale of the experiment is prohibitive for performing LC at every pixel location.

Another means to address chemical complexity is ion mobility spectrometry, a technology that allows for gas phase separation of isobaric and isomeric species based on their size, shape, and charge. ${ }^{30,31}$ For example, ion mobility can be used to distinguish lipid classes based on the relationship between their ion mobility and $m / z$ values, which arise from innate differences in chemical structure..$^{32-34}$ Because 
separations are performed after ionization and are decoupled from spatial sampling, ion mobility can be readily incorporated into existing imaging MS platforms. ${ }^{35,36}$ Furthermore, rapid ion mobility separations (ms) occur within timescales that are compatible with imaging experiments where many thousands of pixels (each with its own ion mobility separation) are collected during a single experiment. The most extensively used ion mobility platforms are drift tube ion mobility spectrometry $(D T I M S)^{37,38}$, field asymmetric ion mobility spectrometry (FAIMS) ${ }^{39}, 40$, traveling-wave ion mobility spectrometry (TWIMS) ${ }^{41}$, 42, and trapped ion mobility spectrometry (TIMS) ${ }^{43,44}$. Recognizing that each of these ion mobility systems provides distinct advantages and disadvantages, TIMS has provided unrivaled resolving power capabilities of $>200$ and $>300$ for singly and multiply charged ions, respectively. ${ }^{43-46}$ For example, a commercially available timsTOF source configuration allows for MALDI imaging of biological tissues at $10 \mu \mathrm{m}$ spatial resolutions without implementing oversampling. ${ }^{47,48}$ Furthermore, TIMS has been shown to increase the overall peak capacity of an imaging experiment and enable separation of isobaric lipid species not able to be resolved by the time-of-flight (TOF) mass analyzer alone. ${ }^{47-49}$

In the current manuscript, we utilize a combination of silicon nanopost arrays (NAPA) and trapped ion mobility imaging mass spectrometry (TIMS IMS) for high spatial resolution and specificity mapping of neutral lipid classes in tissue. We demonstrate the ability of NAPA to serve as an LDI substrate capable of imaging multiple classes of neutral lipids at $20 \mu \mathrm{m}$ spatial resolution, without oversampling, from rat brain and kidney tissue sections in Q-TOF mode. Additionally, these tissues were imaged by TIMS to assess its capability to separate different neutral lipid classes and to differentiate neutral lipid isomers not able to be distinguished using other IMS platforms.

\section{Materials and Methods}

\section{NAPA Imaging Wafers}

The nanofabrication process for producing NAPA imaging wafers has been described previously. ${ }^{50,51}$ Briefly, NAPA imaging wafers were produced from low-resistivity p-type silicon wafers using deepultraviolet projection photolithography followed by deep reactive ion etching. Fabricated nanoposts had final dimensions of $1100 \mathrm{~nm}$ in height, $150 \mathrm{~nm}$ in diameter, with a periodicity of $350 \mathrm{~nm}$.

\section{Sample Preparation}


Intact rat brain and kidney tissue samples were purchased from BiolVT (Westbury, NY, USA) and stored at $-80^{\circ} \mathrm{C}$ until ready for analysis. Tissue samples were cryosectioned with a $10 \mu \mathrm{m}$ thickness using a CM3050 S LEICA cryostat (Wetzlar, Germany) operated between -20 and $-24^{\circ} \mathrm{C}$. Tissue sections were immediately thaw-mounted onto NAPA imaging chips and placed in a desiccator for $\sim 30$ min before IMS analysis.

\section{Trapped Ion Mobility Mass Spectrometry}

All IMS data were collected in positive ion mode on a prototype MALDI timsTOF Pro ${ }^{47}$ (Bruker Daltonics, Bremen, Germany) equipped with a SmartBeam 3D laser (frequency tripled Nd:YAG; $\lambda=355 \mathrm{~nm}$ ) operated at $1 \mathrm{kHz}$ with 14 laser shots per pixel at $78 \%$ and $85 \%$ total laser power for brain and kidney, respectively. TIMS IMS data was collected with beam scan on using a setting of $18 \mu \mathrm{m}$ beam scan size and a $30 \mu \mathrm{m}$ pitch for kidney and $30 \mu \mathrm{m}$ beam scan size and a $40 \mu \mathrm{m}$ pitch for brain. For TIMS analysis, the electric field gradient (EFG) scan time was set at $400 \mathrm{~ms}$ with a mobility $\left(1 / \mathrm{K}_{0}\right)$ range of $0.7-1.7(\mathrm{~V} \cdot \mathrm{s}) / \mathrm{cm}^{2}$ and a mass range of $m / z$ 400-1200. For IMS data collected in Q-TOF only mode, the laser power was set to $90 \%$ and the beam scan was deactivated. The global attenuator setting was set to $20 \%$ for all experiments. Chemical images were visualized using SCiLS Lab Version 2019 (Bruker Daltonics, Bremen, Germany) for all Q-TOF only IMS data with a mass tolerance of $\pm 6.5 \mathrm{mDa}$. TIMS IMS data were processed and visualized using inhouse developed software tools.

Lipid identifications for profiling TIMS experiments analyzing rat kidney and rat brain tissue sections were assigned by exporting out spectra from regions of interest from the ion mobility heatmap and imported into mMass, where spectra were peak picked and deisotoped..$^{52}$ Selected $m / z$ values were then run against an in-house reference list comprised primarily from LipidMaps (lipidmaps.org) with a mass error of \pm 5 ppm.

\section{FT-ICR Mass Spectrometry}

To aid in identification of detected lipid species and in differentiation of isomeric and isobaric lipid species, a parallel analysis was performed on a 15T solariX FT-ICR mass spectrometer (Bruker Daltonics, Bremen, Germany) coupled to an Apollo II ESI-MALDI dual ion source. The instrument was operated from $\mathrm{m} / \mathrm{z} 250$ 1400 with a 1.8 s transient, resulting in an 350,000 mass resolving power at $m / z 686$.

To shift the cationization of lipids from potassiated to sodiated adducts to facilitate collection of tandem MS data, tissue sections were washed $3 \times$ in a chilled $\left(4^{\circ} \mathrm{C}\right) 150 \mathrm{mM}$ ammonium formate aqueous solution for $30 \mathrm{~s}$ to remove sodium and potassium based salts. ${ }^{53}$ Washed tissues were then immediately dried in a desiccator for $\sim 20$ minutes, followed coating with a mixture of sodium carbonate and sodium acetate to 
drive the formation of sodiated lipid adducts. ${ }^{22}$ Precursor $m / z$ values were isolated using a \pm 2 Da window and fragmented using collision induced dissociation with a normalized collision energy ranging from 20$35 \%$.

\section{Data Processing}

TIMS IMS data were exported into a custom binary format optimized for storage and speed of analysis of the ion mobility-IMS data. Each frame/pixel contains between 10,000 and 100,000 centroid peaks, spanning an acquisition range of $m / z 300-1,500$ and $1 / K_{0} 0.8-1.7(\mathrm{~V} \cdot \mathrm{s}) / \mathrm{cm}^{2}$ with 270,861 and 3,312 bins in the MS and ion mobility dimensions, respectively. The processing pipeline requires common $\mathrm{m} / \mathrm{z}$ and $1 / K_{0}$ axes, therefore individual centroid peaks were inserted at their corresponding bin positions along the MS and ion mobility dimensions, where missing values were set to zero. Following the conversion process, an averaged mass spectrum was generated, and peak-picked to select the $\sim 400$ most intense ions. Extracted values were then used to generate ion mobility specific molecular images. Each of the extracted ion mobility-ion images was peak-picked (in the ion mobility dimension) and automatically fitted with Gaussian distributions to identify single and multi-conformational species. Ion mobility-selected ion images were then visualized to examine the conformational-specific localization in the spatial domain.

\section{Results and Discussion}

High Spatial Resolution Tissue Imaging using NAPA-LDI. Rat brain tissue was analyzed to determine the spatial resolution limits of NAPA-LDI IMS without oversampling. Using a laser fluence necessary to produce optimal ion yields, a scanning electron microscope (SEM) image of the ablation area showed the complete removal of the tissue and a crater size of approximately $10 \mu \mathrm{m} \times 12 \mu \mathrm{m}$ with an additional 1-2 $\mu \mathrm{m}$ rim effect (Figure S1). To avoid sampling rim material, subsequent imaging was performed using a 20 $\mu \mathrm{m}$ raster pitch. This represents a significant increase in spatial resolution compared to previous tissue imaging experiments conducted on NAPA. Previous studies were limited to a $100 \mu \mathrm{m}$ spatial resolution without oversampling or $40 \mu \mathrm{m}$ with oversampling. ${ }^{24-26,54,55}$ This higher resolution performance is attributed to the improved focus and higher achievable fluence in our work as compared to these prior experiments.

NAPA imaging of rat brain tissue at $20 \mu \mathrm{m}$ spatial resolution allowed for distinct localization of lipid classes such as HexCer, phosphatidylethanolamine (PE), and phosphatidylethanolamine plasmologen (PEp) to specific anatomical regions of the tissue (Figure 1). For example, $m / z 784.463$ (Figure 1a), provisionally 
identified as $[\mathrm{PE}(36: 4 \mathrm{p})+\mathrm{Na}+\mathrm{K}-\mathrm{H}]^{+}(-2.5 \mathrm{ppm}$ error), was found to be primarily localized to the molecular layer of the cerebellum as well as the stratum pyramidal layer and dentate gyrus-granular layer. Conversely, $m / z 830.507$ (Figure 1 b), provisionally identified as [PE(38:3)+Na+K-H] ${ }^{+}(0.4$ ppm error), was found to be primarily localized to the granular layer, molecular layer, and cerebral cortex. The ion at $m / z$ 848.642 (Figure 1c), provisionally identified as [HexCer(d42:2)+K] $]^{+}(-4.7 \mathrm{ppm}$ error) was found to be primarily localized to the white matter tracts within the cerebellum and corpus callosum. The averaged mass spectrum can be found in Figure 1e. Note, ions with multiple cationic adducts have been shown to commonly form as a result of ionization using NAPA for metabolites and lipids. ${ }^{24,51}$

To further evaluate other tissue types, a rat kidney tissue section was imaged at a $20 \mu \mathrm{m}$ spatial resolution (Figure 2). The averaged mass spectrum can be found in Figure S2. Neutral lipid classes such as TGs, CEs, and HexCers were ionized on the NAPA substrate showing unique spatial distributions within the kidney tissue. For example, $m / z 687.546$ (Figure $2 c$ ), provisionally identified as [CE(18:2)+K] $]^{+}(2.9$ ppm error), was found to be primarily localized to the cortex and outer medulla. In contrast, $m / z 850.652$ (Figure $2 d$ ), provisionally identified as [HexCer(d42:1)+K] $]^{+}(1.2 \mathrm{ppm}$ error), was found to localize specifically to the glomeruli of the cortex, inner medulla, and renal papilla. The ion at $m / z 869.697$ (Figure 2e), provisionally identified as $[\mathrm{TG}(50: 2)+\mathrm{K}]^{+}$(3.4 ppm error) was found to be primarily localized to the adipose tissue surrounding the renal pelvis, and to a lesser extent the cortex.

The capability of NAPA-LDI to selectively ionize classes of neutral lipids (CEs, HexCers, and TGs) eliminates the need for implementation of costly source modifications or specialized sample preparation protocols. The mapping of neutral lipid species is critical for understanding the molecular integrity of the kidney in both health and disease. For example, increased levels of TGs in human plasma has been shown to be a risk factor in humans with chronic kidney disease (CKD), however little is known about the localization of these species to specific functional tissue units in the kidney. ${ }^{56}$ Furthermore, increased levels of HexCers in the renal cortex have been associated with cisplatin-induced acute kidney injury (AKI), an undesirable side effect of cancer treatment. ${ }^{57}$ The ability to map neutral lipids with good sensitivity in kidney tissue would be helpful in determining the molecular underpinnings of these complex diseases.

NAPA-LDI TIMS. TIMS profiling experiments collected from the cortex region of a rat kidney tissue section (Figure 3) showed separation of several different lipid classes in the ion mobility- $m / z$ heatmap. The distinct trendlines of interest are annotated with different colored ellipses (Figure 3a). To identify which lipid classes are represented in these distinct trendlines, spectra were extracted from each region and compared against an in-house reference lipid list with a mass tolerance of $\pm 5 \mathrm{ppm}$. It was determined 
that the three annotated trendlines consisted primarily of CEs (red ellipse, $m / z$ 640-745, 1/ $\mathrm{K}_{0} \sim 1.37-1.44$ $(\mathrm{V} \cdot \mathrm{s}) / \mathrm{cm}^{2}$ ), phospholipids (PCs, PEs, PAs) and HexCers (green ellipse, $\mathrm{m} / \mathrm{z}$ 730-855, 1/ $\mathrm{K}_{0} \sim 1.32-1.42$ $\left.(\mathrm{V} \cdot \mathrm{s}) / \mathrm{cm}^{2}\right)$, and TGs (blue ellipse, $\mathrm{m} / \mathrm{z}$ 840-930, 1/Ko $\left.\sim 1.50-1.60(\mathrm{~V} \cdot \mathrm{s}) / \mathrm{cm}^{2}\right)$. Their corresponding mass spectra can be found in Figures $3 \mathrm{~b}-\mathrm{d}$ along with a subsequent plot of the number of species of each lipid class detected within each trendline. (Figure 4e). Additionally, tandem MS data (Figure S3) was collected for $m / z 671.573$, identified as [CE(18:2)+Na] ${ }^{+}$, and $m / z 879.740$, identified as [TG(52:3)+Na] ${ }^{+}$, to confirm the presence of these neutral lipid classes. Lastly, TIMS data were also collected from profiling experiments for both white matter and gray matter regions of a rat brain tissue section (Figure S4). With respect to white matter, HexCers were the primary lipid class ionized by NAPA with ion mobility values ranging from $1 / \mathrm{K}_{0} \sim 1.45-1.60(\mathrm{~V} \cdot \mathrm{s}) / \mathrm{cm}^{2}$. In contrast, phospholipids such as PEs and PEps dominated the mass spectrum for the gray matter, with ion mobility values ranging from $\sim 1.35-1.45(\mathrm{~V} \cdot \mathrm{s}) / \mathrm{cm}^{2}$. Tandem MS data for $m / z$ 850.673, identified as [HexCer(t42:1)+Na] $]^{+}$, and $m / z$ 836.517, identified as [PE(40:6)+2Na$\mathrm{H}]^{+}$, detected from white matter and gray matter, respectively can be found in Figure S5.

To demonstrate the ability to image neutral lipid isomers generated from a rat kidney tissue section mounted on a NAPA substrate, a TIMS experiment was done (Figure 4). The peak capacity, a measure of the total number of spectral features, was found to increase from 397 in Q-TOF only mode ( $\mathrm{m} / \mathrm{z}$ features) to 772 with TIMS activated (ion mobility- $\mathrm{m} / \mathrm{z}$ features). This is an $~ 95 \%$ increase in detected molecular features for NAPA-LDI IMS of rat kidney tissue. As seen in Figure 4, $m / z 686.585$ was partially resolved into two separate peaks in the ion mobilogram (Figure 4a) with mobility values $1 / \mathrm{K}_{0} 1.30-1.31(\mathrm{~V} \cdot \mathrm{s}) / \mathrm{cm}^{2}$ (purple) and $1 / \mathrm{K}_{0} 1.32-1.34(\mathrm{~V} \cdot \mathrm{s}) / \mathrm{cm}^{2}$ (teal). The ion mobility range $1 / \mathrm{K}_{0} 1.30-1.31(\mathrm{~V} \cdot \mathrm{s}) / \mathrm{cm}^{2}$ (purple) was localized primarily to the outer cortex, whereas ion mobility range $1 / \mathrm{K}_{0} 1.32-1.34(\mathrm{~V} \cdot \mathrm{s}) / \mathrm{cm}^{2}$ (teal) was found to be localized primarily to the inner cortex (Figure $4 \mathrm{~b}-\mathrm{c}$ ). To determine whether this ion mobility separation was a result of resolving isomeric or isobaric lipid species, complementary data were collected on a 15T FT-ICR with a mass resolving power of $\sim 350,000$ at $m / z 686.585$. As seen in Figure S6, there appear to be no neighboring isobaric peaks at $m / z 686.585$ suggesting that the observed species in the TIMS IMS experiment are mobility resolved isomeric species. Based on the tentative identification from mass accuracy, these ions are most likely structural isomers of [Cer(d42:2)+K]+ $(0.3 \mathrm{ppm}$ error).

Ceramides, a class of neutral sphingolipids, have been implicated in a host of debilitating human diseases, including Alzheimer's Disease ${ }^{58}$, Parkinson's Disease ${ }^{59}$, as well as metabolic disorders such as type 2 diabetes and atherosclerosis. ${ }^{60}$ Given the implications that ceramides play in various diseases, the ability to investigate their spatial distributions within biological tissues, as well as the ability to differentiate their 
structural isomers, remains a critical challenge for furthering our understanding of their involvement in healthy and diseased tissues. The combination of the enhanced ionization efficiency for neutral lipid species afforded by NAPA-LDI and the increased molecular coverage, sensitivity, and ability to quickly resolve isobaric and isomeric lipid species afforded by TIMS, provides a unique opportunity for addressing this challenge.

\section{Conclusion}

We have demonstrated the capability of NAPA to image multiple classes of neutral lipids (e.g. CEs, HexCers, and TGs) from biological tissue sections at high spatial resolution ( $20 \mu \mathrm{m})$. This work provides a 5 -fold increase in spatial resolution compared to previous imaging studies performed on NAPA. With further improvements to the laser source optics, or further modifications to the NAPA-LDI substrate permitting use of lower laser fluences (i.e. smaller spot sizes), high spatial resolution imaging applications of biological tissues approaching the single cell level $(\leq 10 \mu \mathrm{m})$ could soon be achieved. Furthermore, the high ion mobility resolving power afforded by TIMS technology enables separation and mapping of different neutral lipid classes. With respect to imaging of rat kidney tissue, the overall peak capacity was increased from 397 (Q-TOF only) to 772 with TIMS enabled, resulting in an 95\% increase in detected molecular features using NAPA-LDI TIMS. This will allow investigation of changes in spatial distributions of isomeric and isobaric lipid species in healthy and diseased tissues. Although there are other technologies that have been developed to increase ionization efficiency of neutral lipids, NAPA-LDI offers the advantage of accessing these different lipid species without additional modifications to the mass spectrometry source or additional sample preparation methods.

\section{Acknowledgements}

The authors would like to acknowledge Biljana Stamenic, Demis D. John, and Brian Thibeault from the University of California, Santa Barbara Nanofabrication Facility for their contributions in the fabrication of NAPA. Scanning electron microscopy measurements were performed at the Vanderbilt Institute of Nanoscale Science and Engineering. Support for these studies was provided by and The National Institutes of Health (NIH) National Institute of General Medical Sciences (P41 GM103391 awarded to R.M.C.), the NIH Common Fund and National Institute of Diabetes, Digestive and Kidney Diseases (U54DK120058 awarded to J.M.S. and R.M.C.) and the NIH Common Fund and National Eye Institute (U54EY032442 
awarded to J.M.S. and R.M.C.). The prototype MALDI timsTOF MS was developed as part of the National Science Foundation Major Research Instrument Program (CBET - 1828299 awarded to J.M.S. and R.M.C.) and the Bruker 15T solariX FT-ICR MS in the Mass Spectrometry Research Center at Vanderbilt University was acquired through the NIH Shared Instrumentation Grant Program (1S100D012359 awarded to R.M.C.). 
Figures:
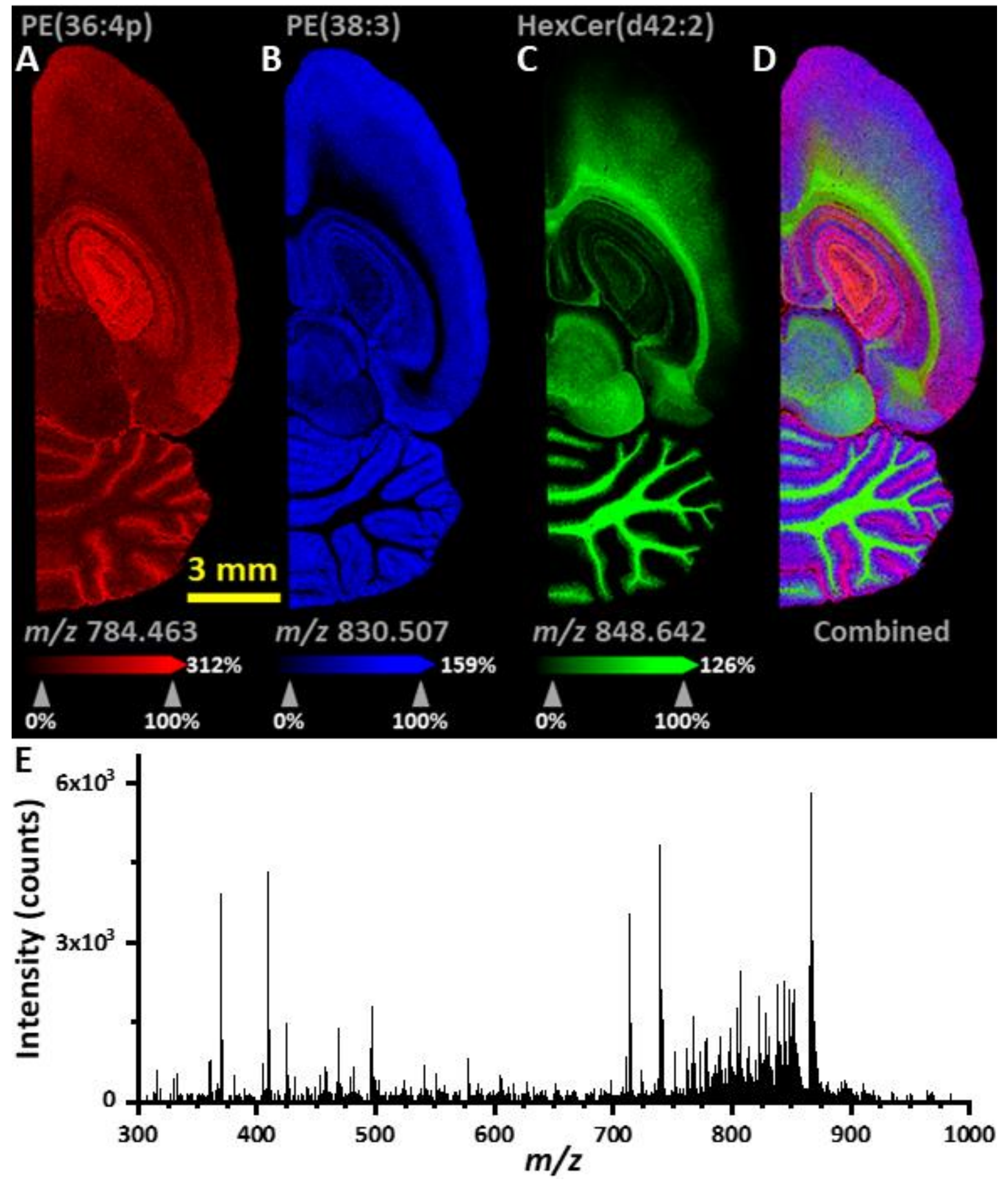

Figure 1. NAPA-LDI-IMS of a rat brain tissue section imaged at $20 \mu \mathrm{m}$ spatial resolution. Chemical images of (A) $P E(36: 4 p),(B) P E(38: 3)$, (C) HexCer(d42:2), and (D) composite of (A)-(D) illustrating the varying spatial distributions detected throughout the tissue. Lipid identifications were assigned based on $\leq 5$ ppm mass error. (E) Averaged mass spectrum of imaged rat brain tissue. 


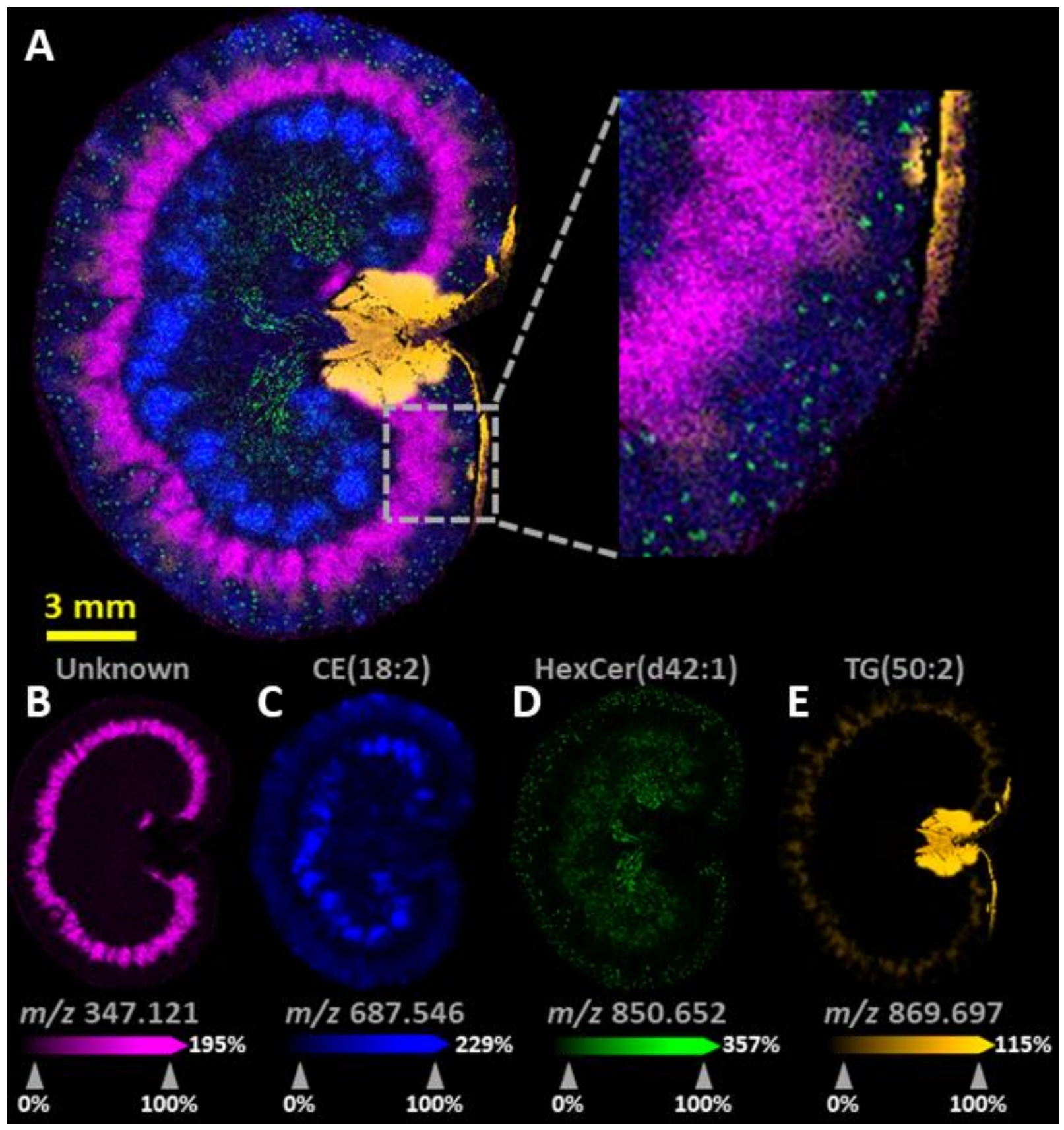

Figure 2. (A) Composite image of select $m / z$ values from NAPA-LDI-IMS of a rat kidney tissue section imaged at $20 \mu \mathrm{m}$ spatial resolution. Chemical images of (B) unknown, (C) CE(18:2), (D) HexCer(d42:1), and $(E) T G(50: 2)$ with varying spatial distributions detected throughout the tissue. Lipid identifications were assigned based on $\leq 5 \mathrm{ppm}$ mass error. 

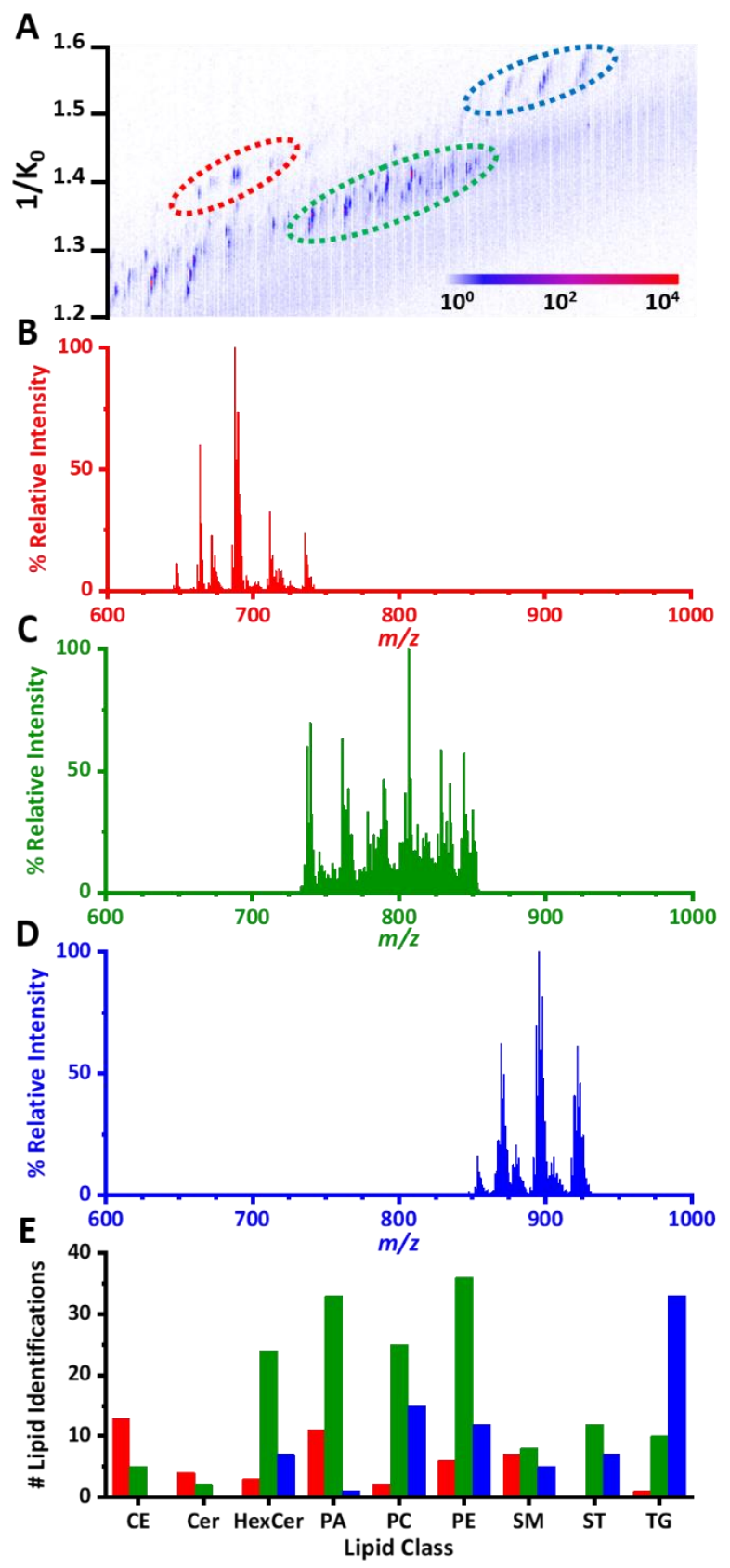

Figure 3. (A) Ion mobility heatmap generated from TIMS analysis of cortex region from rat kidney tissue section. Extracted mass spectra (B) lipid species primarily identified as CEs (red ellipse), (C) lipid species primarily identified as phospholipids and HexCers (green ellipse), and (D) lipid species primarily identified as TGs (blue ellipse) correspond to color-matched ellipses found in (A), highlighting the separation of multiple neutral lipid classes, as well as phospholipids. (E) Bar graph illustrating the lipid class breakdown contained within the different colored ellipses. Bar graph colors are matched with their corresponding ellipse colors. 

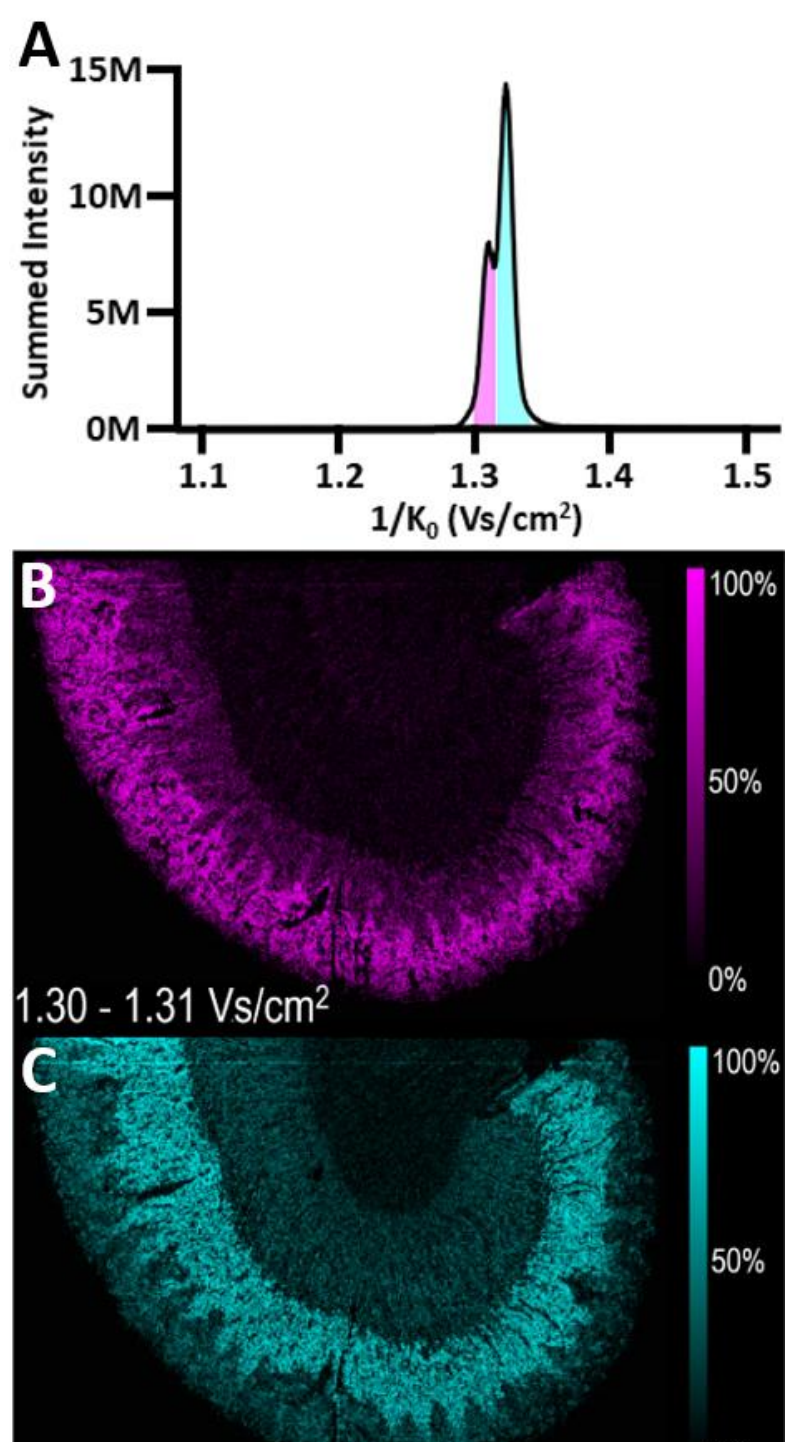

$0 \%$

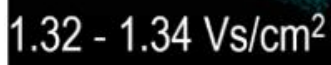

$0 \%$

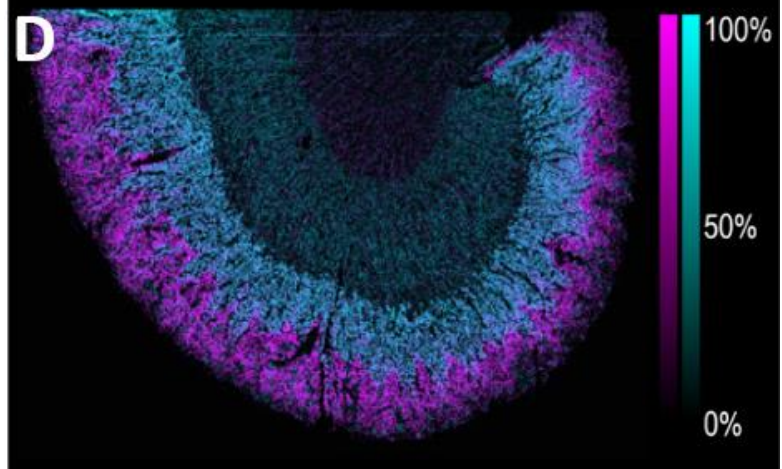

Figure 4. (A) Extracted ion mobilogram for $m / z 686.585$ detected from NAPA-LDI-IMS of rat kidney tissue with TIMS activated. (B) spatial distribution of $m / z 686.585$ with mobility value $1 / \mathrm{K}_{0} 1.30-1.31$ $(\mathrm{V} \cdot \mathrm{s}) / \mathrm{cm}^{2}$ (purple). (C) spatial distribution of $\mathrm{m} / \mathrm{z} 686.585$ with mobility value $1 / \mathrm{K}_{0} 1.32-1.34(\mathrm{~V} \cdot \mathrm{s}) / \mathrm{cm}^{2}$ (teal). (D) Composite image of (B) and (C). 


\section{References:}

1. Caprioli, R. M.; Farmer, T. B.; Gile, J., Molecular imaging of biological samples: Localization of peptides and proteins using MALDI-TOF MS. Analytical Chemistry 1997, 69 (23), 4751-4760.

2. $\quad$ Spraggins, J. M.; Rizzo, D. G.; Moore, J. L.; Rose, K. L.; Hammer, N. D.; Skaar, E. P.; Caprioli, R. M., MALDI FTICR IMS of Intact Proteins: Using Mass Accuracy to Link Protein Images with Proteomics Data. Journal of the American Society for Mass Spectrometry 2015, 26 (6), 974-985.

3. Andersson, M.; Groseclose, M. R.; Deutch, A. Y.; Caprioli, R. M., Imaging mass spectrometry of proteins and peptides: 3D volume reconstruction. Nature Methods 2008, 5 (1), 101-108.

4. $\quad$ Buchberger, A. R.; Vu, N. Q.; Johnson, J.; DeLaney, K.; Li, L. J., A Simple and Effective Sample Preparation Strategy for MALDI-MS Imaging of Neuropeptide Changes in the Crustacean Brain Due to Hypoxia and Hypercapnia Stress. Journal of the American Society for Mass Spectrometry 2020, 31 (5), 1058-1065.

5. Murphy, R. C.; Hankin, J. A.; Barkley, R. M., Imaging of lipid species by MALDI mass spectrometry. Journal of Lipid Research 2009, 50, S317-S322.

6. $\quad$ Anderson, D. M. G.; Ablonczy, Z.; Koutalos, Y.; Spraggins, J.; Crouch, R. K.; Caprioli, R. M.; Schey, K. L., High Resolution MALDI Imaging Mass Spectrometry of Retinal Tissue Lipids. Journal of the American Society for Mass Spectrometry 2014, 25 (8), 1394-1403.

7. Wiseman, J. M.; Ifa, D. R.; Zhu, Y. X.; Kissinger, C. B.; Manicke, N. E.; Kissinger, P. T.; Cooks, R. G., Desorption electrospray ionization mass spectrometry: Imaging drugs and metabolites in tissues. Proceedings of the National Academy of Sciences of the United States of America 2008, 105 (47), 1812018125.

8. Nemes, P.; Woods, A. S.; Vertes, A., Simultaneous Imaging of Small Metabolites and Lipids in Rat Brain Tissues at Atmospheric Pressure by Laser Ablation Electrospray lonization Mass Spectrometry. Analytical Chemistry 2010, 82 (3), 982-988.

9. Aichler, M.; Walch, A., MALDI Imaging mass spectrometry: current frontiers and perspectives in pathology research and practice. Laboratory Investigation 2015, 95 (4), 422-431.

10. Eberlin, L. S.; Norton, I.; Dill, A. L.; Golby, A. J.; Ligon, K. L.; Santagata, S.; Cooks, R. G.; Agar, N. Y. R., Classifying Human Brain Tumors by Lipid Imaging with Mass Spectrometry. Cancer Research 2012, $72(3), 645-654$.

11. Guenther, S.; Muirhead, L. J.; Speller, A. V. M.; Golf, O.; Strittmatter, N.; Ramakrishnan, R.; Goldin, R. D.; Jones, E.; Veselkov, K.; Nicholson, J.; Darzi, A.; Takats, Z., Spatially Resolved Metabolic Phenotyping of Breast Cancer by Desorption Electrospray Ionization Mass Spectrometry. Cancer Research 2015, 75 (9), 1828-1837.

12. Goodwin, R. J. A.; Takats, Z.; Bunch, J., A Critical and Concise Review of Mass Spectrometry Applied to Imaging in Drug Discovery. Slas Discovery 2020, 25 (9), 963-976.

13. Swales, J. G.; Hamm, G.; Clench, M. R.; Goodwin, R. J. A., Mass spectrometry imaging and its application in pharmaceutical research and development: A concise review. International Journal of Mass Spectrometry 2019, 437, 99-112.

14. Norris, J. L.; Caprioli, R. M., Analysis of Tissue Specimens by Matrix-Assisted Laser Desorption/Ionization Imaging Mass Spectrometry in Biological and Clinical Research. Chemical Reviews 2013, 113 (4), $2309-2342$.

15. Korte, A. R.; Yandeau-Nelson, M. D.; Nikolau, B. J.; Lee, Y. J., Subcellular-level resolution MALDIMS imaging of maize leaf metabolites by MALDI-linear ion trap-Orbitrap mass spectrometer. Analytical and Bioanalytical Chemistry 2015, 407 (8), 2301-2309. 
16. Kompauer, M.; Heiles, S.; Spengler, B., Atmospheric pressure MALDI mass spectrometry imaging of tissues and cells at 1.4-mu m lateral resolution. Nature Methods 2017, 14 (1), 90-96.

17. Zavalin, A.; Todd, E. M.; Rawhouser, P. D.; Yang, J. H.; Norris, J. L.; Caprioli, R. M., Direct imaging of single cells and tissue at sub-cellular spatial resolution using transmission geometry MALDI MS. Journal of Mass Spectrometry 2012, 47 (11), 1473-1481.

18. Niehaus, M.; Soltwisch, J.; Belov, M. E.; Dreisewerd, K., Transmission-mode MALDI-2 mass spectrometry imaging of cells and tissues at subcellular resolution. Nature Methods 2019, 16 (9), 925-+. 19. Spivey, E. C.; McMillen, J. C.; Ryan, D. J.; Spraggins, J. M.; Caprioli, R. M., Combining MALDI-2 and transmission geometry laser optics to achieve high sensitivity for ultra-high spatial resolution surface analysis. Journal of Mass Spectrometry 2019, 54 (4), 366-370.

20. Dufresne, M.; Masson, J. F.; Chaurand, P., Sodium-Doped Gold-Assisted Laser Desorption Ionization for Enhanced Imaging Mass Spectrometry of Triacylglycerols from Thin Tissue Sections. Analytical Chemistry 2016, 88 (11), 6018-6025.

21. Jackson, S. N.; Baldwin, K.; Muller, L.; Womack, V. M.; Schultz, J. A.; Balaban, C.; Woods, A. S., Imaging of lipids in rat heart by MALDI-MS with silver nanoparticles. Analytical and Bioanalytical Chemistry 2014, 406 (5), 1377-1386.

22. Dufresne, M.; Patterson, N. H.; Norris, J. L.; Caprioli, R. M., Combining Salt Doping and Matrix Sublimation for High Spatial Resolution MALDI Imaging Mass Spectrometry of Neutral Lipids. Analytical Chemistry 2019, 91 (20), 12928-12934.

23. Bowman, A. P.; Bogie, J. F. J.; Hendriks, J. J. A.; Haidar, M.; Belov, M.; Heeren, R. M. A.; Ellis, S. R., Evaluation of lipid coverage and high spatial resolution MALDI-imaging capabilities of oversampling combined with laser post-ionisation. Analytical and Bioanalytical Chemistry 2020, 412 (10), 2277-2289.

24. Fincher, J. A.; Dyer, J. E.; Korte, A. R.; Yadavilli, S.; Morris, N. J.; Vertes, A., Matrix-free mass spectrometry imaging of mouse brain tissue sections on silicon nanopost arrays. Journal of Comparative Neurology 2019, 527 (13), 2101-2121.

25. Fincher, J. A.; Korte, A. R.; Dyer, J. E.; Yadavilli, S.; Morris, N. J.; Jones, D. R.; Shanmugam, V. K.; Pirlo, R. K.; Vertes, A., Mass spectrometry imaging of triglycerides in biological tissues by laser desorption ionization from silicon nanopost arrays. Journal of Mass Spectrometry 2020, 55 (4).

26. Fincher, J. A.; Jones, D. R.; Korte, A. R.; Dyer, J. E.; Parlanti, P.; Popratiloff, A.; Brantner, C. A.; Morris, N. J.; Pirlo, R. K.; Shanmugam, V. K.; Vertes, A., Mass Spectrometry Imaging of Lipids in Human Skin Disease Model Hidradenitis Suppurativa by Laser Desorption lonization from Silicon Nanopost Arrays. Scientific Reports 2019, 9, 17508.

27. Quehenberger, O.; Armando, A. M.; Brown, A. H.; Milne, S. B.; Myers, D. S.; Merrill, A. H.; Bandyopadhyay, S.; Jones, K. N.; Kelly, S.; Shaner, R. L.; Sullards, C. M.; Wang, E.; Murphy, R. C.; Barkley, R. M.; Leiker, T. J.; Raetz, C. R. H.; Guan, Z. Q.; Laird, G. M.; Six, D. A.; Russell, D. W.; McDonald, J. G.; Subramaniam, S.; Fahy, E.; Dennis, E. A., Lipidomics reveals a remarkable diversity of lipids in human plasma. Journal of Lipid Research 2010, 51 (11), 3299-3305.

28. Han, X. L.; Gross, R. W., Shotgun lipidomics: Electrospray ionization mass spectrometric analysis and quantitation of cellular lipidomes directly from crude extracts of biological samples. Mass Spectrometry Reviews 2005, 24 (3), 367-412.

29. Shevchenko, A.; Simons, K., Lipidomics: coming to grips with lipid diversity. Nature Reviews Molecular Cell Biology 2010, 11 (8), 593-598.

30. Wu, Q.; Wang, J. Y.; Han, D. Q.; Yao, Z. P., Recent advances in differentiation of isomers by ion mobility mass spectrometry. Trac-Trends in Analytical Chemistry 2020, 124.

31. May, J. C.; McLean, J. A., Ion Mobility-Mass Spectrometry: Time-Dispersive Instrumentation. Analytical Chemistry 2015, 87 (3), 1422-1436. 
32. Harris, R. A.; Leaptrot, K. L.; May, J. C.; McLean, J. A., New frontiers in lipidomics analyses using structurally selective ion mobility-mass spectrometry. Trac-Trends in Analytical Chemistry 2019, 116, 316-323.

33. Leaptrot, K. L.; May, J. C.; Dodds, J. N.; McLean, J. A., Ion mobility conformational lipid atlas for high confidence lipidomics. Nature Communications 2019, 10.

34. Kyle, J. E.; Zhang, X.; Weitz, K. K.; Monroe, M. E.; Ibrahim, Y. M.; Moore, R. J.; Cha, J.; Sun, X. F.; Lovelace, E. S.; Wagoner, J.; Polyak, S. J.; Metz, T. O.; Dey, S. K.; Smith, R. D.; Burnum-Johnson, K. E.; Baker, E. S., Uncovering biologically significant lipid isomers with liquid chromatography, ion mobility spectrometry and mass spectrometry. Analyst 2016, 141 (5), 1649-1659.

35. Rivera, E. S.; Djambazova, K. V.; Neumann, E. K.; Caprioli, R. M.; Spraggins, J. M., Integrating ion mobility and imaging mass spectrometry for comprehensive analysis of biological tissues: A brief review and perspective. Journal of Mass Spectrometry 2020, 55 (12).

36. Sans, M.; Feider, C. L.; Eberlin, L. S., Advances in mass spectrometry imaging coupled to ion mobility spectrometry for enhanced imaging of biological tissues. Current Opinion in Chemical Biology 2018, 42, 138-146.

37. May, J. C.; Goodwin, C. R.; Lareau, N. M.; Leaptrot, K. L.; Morris, C. B.; Kurulugama, R. T.; Mordehai, A.; Klein, C.; Barry, W.; Darland, E.; Overney, G.; Imatani, K.; Stafford, G. C.; Fjeldsted, J. C.; McLean, J. A., Conformational Ordering of Biomolecules in the Gas Phase: Nitrogen Collision Cross Sections Measured on a Prototype High Resolution Drift Tube Ion Mobility-Mass Spectrometer. Analytical Chemistry 2014, 86 (4), 2107-2116.

38. Valentine, S. J.; Koeniger, S. L.; Clemmer, D. E., A split-field drift tube for separation and efficient fragmentation of biomolecular ions. Analytical Chemistry 2003, 75 (22), 6202-6208.

39. Kolakowski, B. M.; Mester, Z., Review of applications of high-field asymmetric waveform ion mobility spectrometry (FAIMS) and differential mobility spectrometry (DMS). Analyst 2007, 132 (9), 842864.

40. Purves, R. W.; Guevremont, R., Electrospray ionization high-field asymmetric waveform ion mobility spectrometry-mass spectrometry. Analytical Chemistry 1999, 71 (13), 2346-2357.

41. Shvartsburg, A. A.; Smith, R. D., Fundamentals of Traveling Wave lon Mobility Spectrometry. Analytical Chemistry 2008, 80 (24), 9689-9699.

42. Zhong, Y. Y.; Hyung, S. J.; Ruotolo, B. T., Characterizing the resolution and accuracy of a secondgeneration traveling-wave ion mobility separator for biomolecular ions. Analyst 2011, 136 (17), 35343541.

43. Michelmann, K.; Silveira, J. A.; Ridgeway, M. E.; Park, M. A., Fundamentals of Trapped Ion Mobility Spectrometry. Journal of the American Society for Mass Spectrometry 2015, 26 (1), 14-24.

44. Ridgeway, M. E.; Lubeck, M.; Jordens, J.; Mann, M.; Park, M. A., Trapped ion mobility spectrometry: A short review. International Journal of Mass Spectrometry 2018, 425, 22-35.

45. Hernandez, D. R.; DeBord, J. D.; Ridgeway, M. E.; Kaplan, D. A.; Park, M. A.; Fernandez-Lima, F., lon dynamics in a trapped ion mobility spectrometer. Analyst 2014, 139 (8), 1913-1921.

46. Ridgeway, M. E.; Silveira, J. A.; Meier, J. E.; Park, M. A., Microheterogeneity within conformational states of ubiquitin revealed by high resolution trapped ion mobility spectrometry. Analyst 2015, 14 (20), 6964-6972.

47. Spraggins, J. M.; Djambazova, K. V.; Rivera, E. S.; Migas, L.; Neumann, E. K.; Fuetterer, A.; Suetering, J.; Goedecke, N.; Ly, A.; Van de Plas, R.; Caprioli, R. M., High Performance Molecular Imaging with MALDI Trapped Ion Mobility Time-of-Flight (timsTOF) Mass Spectrometry. Analytical Chemistry 2019.

48. Soltwisch, J.; Heijs, B.; Koch, A.; Vens-Cappell, S.; Höhndorf, J.; Dreisewerd, K., MALDI-2 on a Trapped Ion Mobility Quadrupole Time-of-Flight Instrument for Rapid Mass Spectrometry Imaging and Ion Mobility Separation of Complex Lipid Profiles. Analytical Chemistry 2020, 92 (13), 8697-8703. 
49. Djambazova, K. V.; Klein, D. R.; Migas, L. G.; Neumann, E. K.; Rivera, E. S.; Van de Plas, R.; Caprioli, R. M.; Spraggins, J. M., Resolving the Complexity of Spatial Lipidomics Using MALDI TIMS Imaging Mass Spectrometry. Analytical Chemistry 2020.

50. Morris, N. J.; Anderson, H.; Thibeault, B.; Vertes, A.; Powell, M. J.; Razunguzwa, T. T., Laser desorption ionization (LDI) silicon nanopost array chips fabricated using deep UV projection lithography and deep reactive ion etching. RSC Advances 2015, 5 (88), 72051-72057.

51. Korte, A. R.; Stopka, S. A.; Morris, N.; Razunguzwa, T.; Vertes, A., Large-Scale Metabolite Analysis of Standards and Human Serum by Laser Desorption Ionization Mass Spectrometry from Silicon Nanopost Arrays. Analytical Chemistry 2016, 88 (18), 8989-8996.

52. Strohalm, M.; Kavan, D.; Novak, P.; Volny, M.; Havlicek, V., mMass 3: A Cross-Platform Software Environment for Precise Analysis of Mass Spectrometric Data. Analytical Chemistry 2010, 82 (11), 4648-4651.

53. Angel, P. M.; Spraggins, J. M.; Baldwin, H. S.; Caprioli, R., Enhanced Sensitivity for High Spatial Resolution Lipid Analysis by Negative Ion Mode Matrix Assisted Laser Desorption Ionization Imaging Mass Spectrometry. Analytical Chemistry 2012, 84 (3), 1557-1564.

54. Fincher, J. A.; Korte, A. R.; Yadavilli, S.; Morris, N. J.; Vertes, A., Multimodal imaging of biological tissues using combined MALDI and NAPA-LDI mass spectrometry for enhanced molecular coverage. Analyst 2020, 145 (21), 6910-6918.

55. Stopka, S. A.; Rong, C.; Korte, A. R.; Yadavilli, S.; Nazarian, J.; Razunguzwa, T. T.; Morris, N. J.; Vertes, A., Molecular Imaging of Biological Samples on Nanophotonic Laser Desorption Ionization Platforms. Angew Chem Int Edit 2016, 55 (14), 4482-4486.

56. Dincer, N.; Dagel, T.; Afsar, B.; Covic, A.; Ortiz, A.; Kanbay, M., The effect of chronic kidney disease on lipid metabolism. International Urology and Nephrology 2019, 51 (2), 265-277.

57. Dupre, T. V.; Doll, M. A.; Shah, P. P.; Sharp, C. N.; Siow, D.; Megyesi, J.; Shayman, J.; Bielawska, A.; Bielawski, J.; Beverly, L. J.; Hernandez-Corbacho, M.; Clarke, C. J.; Snider, A. J.; Schnellmann, R. G.; Obeid, L. M.; Hannun, Y. A.; Siskind, L. J., Inhibiting glucosylceramide synthase exacerbates cisplatin-induced acute kidney injury. Journal of Lipid Research 2017, 58 (7), 1439-1452. 58. Jembrek, M. J.; Hof, P. R.; Simic, G., Ceramides in Alzheimer's Disease: Key Mediators of Neuronal Apoptosis Induced by Oxidative Stress and A beta Accumulation. Oxidative Medicine and Cellular Longevity 2015, 2015.

59. Plotegher, N.; Bubacco, L.; Greggio, E.; Civiero, L., Ceramides in Parkinson's Disease: From Recent Evidence to New Hypotheses. Frontiers in Neuroscience 2019, 13.

60. Turpin-Nolan, S. M.; Bruning, J. C., The role of ceramides in metabolic disorders: when size and localization matters. Nature Reviews Endocrinology 2020, 16 (4), 224-233. 